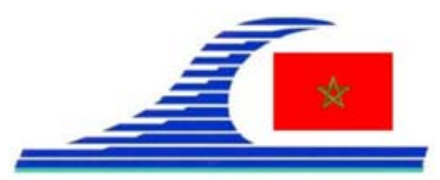

Conférence Méditerranéenne Côtière et Maritime

EDITION 2, TANGER, MAROC (2011)

Coastal and Maritime Mediterranean Conference

Disponible en ligne - http://www.paralia.fr - Available online

\title{
Distribution des sédiments en milieu côtier de la région de Casablanca-Dar Bou Azza (Maroc)
}

\author{
Sana HICHOUR ${ }^{1}$, Rachid ESSAMOUD ${ }^{1}$
}

1. LGSE: Laboratoire de Géoressources sédimentaires et Environnement, Département de Géologie. Faculté des Sciences Ben Msik, B.P. 7955, Casablanca, Maroc.

hichoursana@yahoo.fr

\section{Résumé :}

Une étude sédimentologique des sables actuels du littoral de Casablanca-Dar Bou Azza a été réalisée dans le but de déterminer, d'une part les caractéristiques granulométriques et minéralogiques, et d'autre part les facteurs de contrôle de la distribution et le transport des sédiments. L'analyse granulométrique a montré une prédominance des sables moyens à grossiers en période hivernale et des sables fins en période estivale. Le cortège minéralogique transparent est largement dominé par les minéraux de roches basaltiques et métamorphiques, affleurant dans la meseta côtière. Les marées, les courants, les vagues et le vent sont les agents responsables de la distribution et du transport de ces sédiments.

Mots clés : Littoral - Plage - Sable - Dynamique sédimentaire - Casablanca-Dar Bou Azza - Maroc

\section{Introduction}

La zone côtière de Casablanca-Dar Bou Azza connaît dans sa partie Est une activité anthropique qui endommage la dune bordière. Cependant, la partie Ouest, dépourvue d'aménagement côtier connaît une stabilité et un certain équilibre entre les plages sableuses et les dunes bordières.

Le présent travail est une étude pluridisciplinaire actualisée de la dynamique sédimentaire de la frange côtière Casablanca-Dar Bou Azza. L'objectif est de caractériser ces sables de point de vue granulométrique et minéralogique. Il traite aussi les processus et mécanismes de transport des sables récents, ainsi que les facteurs de contrôle de la distribution granulométrique des faciès.

\section{Cadre géomorphologique et hydrodynamique}

La morphologie du littoral étudié présente un talus de $10 \mathrm{~m}$ d'épaisseur, situé à environ $1 \mathrm{~km}$ de la ligne de rivage actuelle. Ce talus correspond à une falaise morte nommée ouljienne (GIGOUT, 1951); séparant deux parties, une série de cordons littoraux consolidés d'âge pliocène et quaternaire (BEAUDET et al., 1967), et une dépression allongée (Oulja) au pied de cette falaise. Ces cordons dunaires d'âge fini-ouljien (HOURIMECHE, 1999) sont parallèles à la ligne actuelle de rivage (figure 1), et ils se 
La connaissance de la Mer :

un vecteur du développement durable en Méditerranée

succèdent du plus ancien au plus récent de l'arrière pays vers la zone côtière. Une intense dérive littorale a permis l'édification de ces cordons-barrières (ZOURARAH, 2002).

La propagation de la houle du large vers la côte Casablanca-Dar Bou Azza (AIT LAAMEL, 2004) est caractérisée par un croisement des rayons en face de la plage Badiâa et en face de la partie Est de la plage Merezg. Par contre on note une convergence des rayons au niveau de la plage Sidi Abderrahmane, la partie Ouest de la plage Merezg et sur le cap d'Aïn Diab. Ces croisements et convergences sont liés à la présence des hauts fonds et des dorsales sous-marines, ils sont plus accentués en hiver qu'en été. Les rayons restent parallèles entre eux et perpendiculaires à la côte au niveau de la baie d'Aïn Diab et de la plage Rmel.

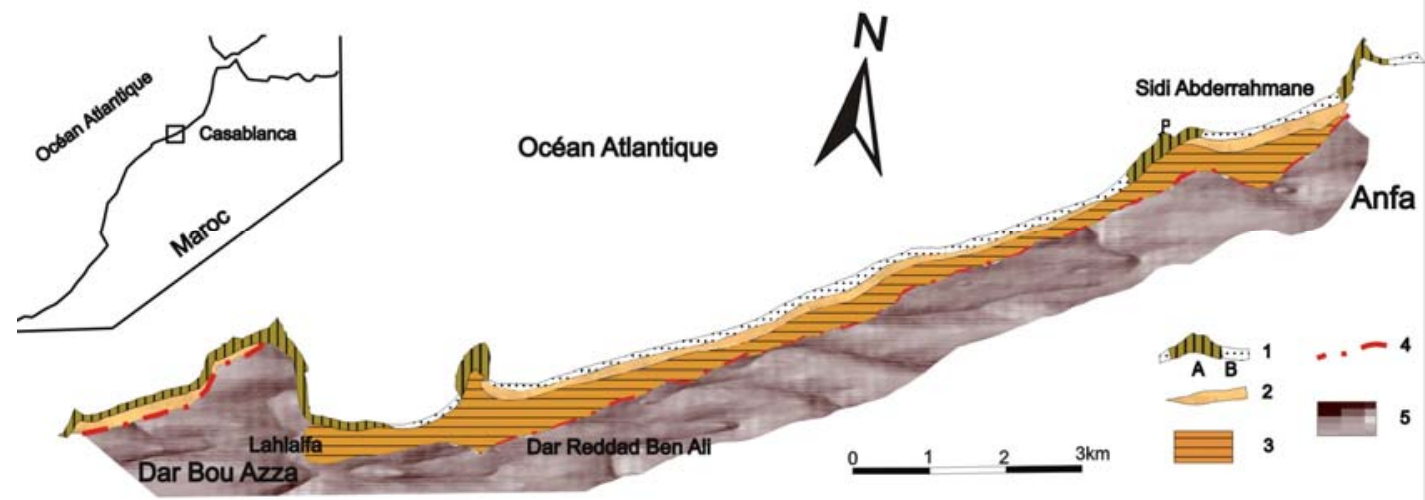

Figure 1. Unités géomorphologiques du littoral Casablanca - Dar Bou Azza, (1A) estran rocheux, (1B) estran sableux, (2) cordon dunaire littoral, (3) dépression ouljienne, (4) falaise morte, (5) plates-formes et cordons anciens.

\section{Méthodologie}

\subsection{L'analyse granulométrique}

L'analyse granulométrique a été effectuée sur des sables bruts (une trentaine d'échantillons ont été prélevées au niveau de l'estran). Pour le tamisage, on a utilisé une série de 18 tamis de la série AFNOR, et les résultats ont été représentés graphiquement sous forme de courbes cumulatives. A partir des formules FOLK et WARD (1957) basées sur l'unité $\Phi$, on a calculé les paramètres de distribution.

\subsection{Mode de transport}

Le mode de transport a été déterminé selon la méthode de PASSEGA (1963), qui se base sur l'interprétation des données granulométriques. Cette méthode permet de définir trois modes de transport qui sont : la suspension, la saltation et le charriage, voir exemple plage Aïn Diab, et plage Merzeg (figure 2). 

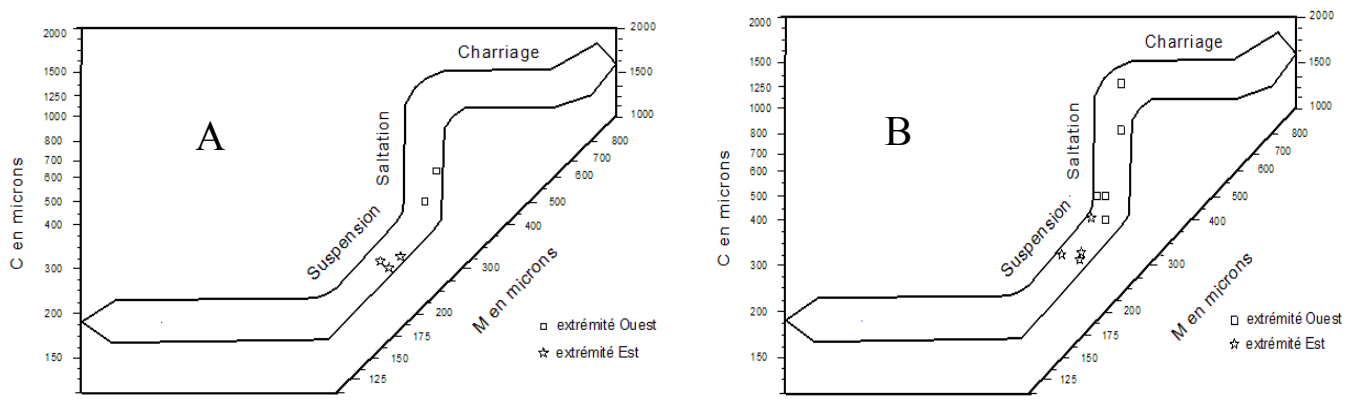

Figure 2. Mode de transport des sables prélevés en été, A) au niveau de la plage Aïn Diab, B) au niveau de la plage Merzeg.

\subsection{Morphoscopie et minéraux lourds}

- L'analyse morphoscopique a été réalisée suivant la méthode de CAILLEUX et TRICART (1959).

- L'étude des minéraux lourds a été réalisée selon la méthode de PARFENOFF et al. (1970). La séparation des minéraux lourds de ceux légers a été faite par le bromoforme de densité $\mathrm{d}=2,89$.

\section{Interprétation et synthèse}

\subsection{Répartition des sables de plages}

L'analyse granulométrique des sables prélevés le long du littoral Casablanca- Dar Bou Azza, pendant les deux périodes, montre un large spectre granulométrique allant des sables fins aux sables grossiers. Les sables moyens et grossiers sont repérés au niveau des plages à platiers rocheux où les vagues convergent, augmentant ainsi l'énergie du milieu (prédominance de l'érosion). Cependant les sables fins sont enregistrés au niveau des zones sableuses séparant ces platiers où les vagues divergent et leur énergie se dissipent.

L'étude de la variation saisonnière de la dynamique sédimentaire montre qu'au niveau de la plage Rmel, les sables sont fins à grossiers. Ceci est dû à la conjonction de la dynamique marine et de la dynamique éolienne qui enrichissent l'estran en sables fins. Cette constatation est confirmée par les proportions égales des grains émoussés-mats et émoussés-luisants.

Les sables de la partie centrale de la plage Bdiaâ, sont plus fins pendant l'hiver que pendant l'été. Ceci est dû à la forte énergie des vagues dans cette partie de la plage entraînant l'alimentation de l'estran par des sables fins de la dune bordière. Dans d'autres zones, notamment au niveau du marabout Sidi Abderrahmane et de la plage de l'embouchure de l'Oued Merzeg, les sables sont plus grossiers en hiver qu'en été. Ceci est dû à la forte agitation hivernale, et s'explique par la présence de pointes rocheuses au niveau de ces zones et par l'absence de dune bordière. 
La connaissance de la Mer :

un vecteur du développement durable en Méditerranée

\subsection{Mode de transport}

L'étude des mécanismes de transport des sables prélevés sur le littoral de CasablancaDar Bou Azza pendant les deux saisons montre deux modes de transport :

- Un mode de transport par saltation et par charriage au niveau des plages à platiers rocheux, qui sont des zones de moyenne à forte agitation.

- Un mode de transport par saltation et suspension au niveau des plages sableuses, qui sont des zones de moyenne à faible agitation.

\subsection{Minéraux lourds}

L'étude des minéraux lourds montre un cortège minéralogique très uniforme, largement dominé par l'augite suivie de l'hornblende. Ces deux minéraux proviennent essentiellement des basaltes triasiques affleurant dans les vallées de l'Oued Mellah et l'Oued Nefifikh. Ces minéraux sont répartis sur la côte par une dérive littorale de direction nord-sud.

\section{Références bibliographiques}

AIT LAAMEL M. (2004). Modélisation numérique de la houle côtière. Thèse de Doctorat d'Etat, Fac. Sci. Ben M'sik, Casablanca, Maroc.

BEAUDET G., MAURER G., RUELLAN A. (1967). Le Quaternaire marocain, observations et hypothèses nouvelles. Rev. Géol. Dyn. Géogr. Phys., t 2, Vol. Tx, Fasc. 4, Paris, pp 269-310.

CAILLEUX A., TRICART J., (1959). Initiation à l'étude des sables et des galets. T. 3, Cent. Docum. Univer. Paris.

FOLK R.L., WARD W.C. (1957). Brazos river bar: a staday in the significance of grain size parameters. J. Sedim. Petrol., 27 (1), pp 3-26.

GIGOUT M. (1951). Etude géologique de la meseta marocaine occidentale (arrière pays de Casablanca, Mazagan et Safi). Notes et Mémoires n ${ }^{\circ} 86, \mathrm{~T} 1$ : Texte, Rabat.

HOURIMECHE A. (1999). Etude sédimentologique des formations plio-quaternaires et des dépôts côtiers actuels de la région d'Essaouira - comparaison avec la région de Casablanca. Thèse Doctorat d'Etat. Fac. Sc. Ben M'Sik, Casablanca ; 271 p.

Thèse 3éme Cycle, Univ. Hassan II-Mohammedia. Fac. Sc. Ben M'sik Maroc ; 227 p.

PARFENOFF A., POMEROL C., TOURENQ J. (1970). Les minéraux en grains : méthodes d'etudes et détermination. Edit. Masson et Cie, Paris VI, $550 \mathrm{p}$.

PASSEGA R. (1963). Analyses granulométriques, outils géologique pratique. Rev. Inst. Fr. pétrol. pp 1489-1499.

ZOURARAH B. (2002). Les processus côtiers actuels et leur impact sur l'environnement littoral des Doukkala, approches hydrodynamique, morphologique et géochimique. Thèse de Doctorat, Univ. Chouaib Dokkali, El Jadida, 228 p. 